\title{
Nutritional Status Assessed by Scored Patient-generated Subjective Global Assessment Associated with Length of Hospital Stay in Adult Patients Receiving an Appendectomy
}

\author{
Tzu-Hao Huang ${ }^{1}$, Ching-Chi Chi ${ }^{2,3}$, Chin-Hua Liu ${ }^{4}$, Cheng-Chih Chang ${ }^{1}$, Liang-Mou Kuo ${ }^{1}$, \\ Ching-Chuan $\mathrm{Hsieh}^{4,5}$
}

Background: Malnutrition has been associated with poor health outcomes in hospitalized patients. This study assessed the validity of the scored patient-generated subjective global assessment (PG-SGA) in adult patients who had undergone an open appendectomy, and examined the association of this assessment tool with length of hospital stay.

Methods: Nutritional status was determined by using the scored PG-SGA in adult patients $(n=86)$ who had undergone an open appendectomy within 24 hours of admission. Variables were compared between well-nourished and malnourished participants. Regression analysis was used to identify potential predictors for length of hospital stay. Receiver operator characteristic (ROC) analysis was used to examine the validity of the PG-SGA score to predict the nutritional status.

Results: On admission, $17 \%$ of the study subjects were malnourished and associated with a significantly older age (53.0 vs. 39.5), greater PG-SGA score (8 vs. 2), higher comorbidity (67\% vs. $27 \%$ ), and longer length of hospital stay $(6.9 \mathrm{~d}$ vs. $4.1 \mathrm{~d})$. The PG-SGA score and comorbidity

At a Glance Commentary

Scientific background of the subject

The incidence of malnutrition is between $15 \%$ and $50 \%$ in hospitalized patients and poor nutrition has been related to poor health outcomes. The aim of this study is to assess the validity of the scored PG-SGA as a nutritional assessment tool in adult patients who had undergone an appendectomy and to examine the association of the scored PG-SGA with length of hospital stay.

What this study adds to the field

According to this study, the scored PG-SGA is an effective tool for assessing the nutritional status of patients with acute appendicitis and is associated with length of hospital stay. were the determined risk factors for length of hospital stay after performing multiple regression analysis. Furthermore, the PG-SGA score had a significantly positive correlation with length of hospital stay (Spearman's rho $=0.378, p<0.001$ ). The area under the ROC curve indicating the PG-SGA score, compared with nutritional status, is 0.9751.

Conclusions: The scored PG-SGA in adults receiving an appendectomy is significantly associated with length of hospital stay, and is an effective tool for assessing the nutritional status of patients with cancer and chronic illness, as well as of patients with acute surgical abdomen.

(Biomed J 2014;37:71-77)

Key words: appendectomy, length of hospital stay, nutritional status, scored patient-generated subjective global assessment

From the ${ }^{1}$ Division of General Surgery, Department of Surgery, Chang Gung Memorial Hospital at Chiayi, Chang Gung University College of Medicine, Taoyuan, Taiwan; ${ }^{2}$ Department of Dermatology, Chang Gung Memorial Hospital at Chiayi, Chang Gung University College of Medicine, Taoyuan, Taiwan; ${ }^{3}$ Centre for Evidence-Based Medicine, Chang Gung Memorial Hospital at Chiayi, Chang Gung University College of Medicine, Taoyuan, Taiwan; ${ }^{4}$ Department of Nutrition Chang Gung Memorial Hospital at Chiayi, Chang Gung University College of Medicine, Taoyuan, Taiwan; ${ }^{5}$ Department of Respiratory Care, Chang Gung University of Science and Technology, Chiayi, Taiwan Received: Sep. 19, 2012; Accepted: Mar. 19, 2013

Correspondence to: Dr. Ching-Chuan Hsieh, Department of Nutrition, Chang Gung Memorial Hospital at Chiayi. 6, W. Sec., Jiapu Rd., Puzi, Chiayi 613, Taiwan (R.O.C.). Tel: 886-5-3621000, ext 3129; Fax: 886-5-3623002; E-mail: jeffrey570404@ gmail.com

DOI: $10.4103 / 2319-4170.113183$ 
$\mathrm{T}_{\mathrm{s}}^{\mathrm{s}}$ The incidence of malnutrition is between $15 \%$ and $50 \%$ in hospitalized patients and poor nutrition has been associated with longer hospital stays, higher complication rates, higher hospital costs, and higher mortality rates. ${ }^{[1-3]}$ Therefore, nutritional status should be accurately evaluated and receive effective intervention to improve the consequences of malnutrition.

The scored patient-generated subjective global assessment (PG-SGA) is a nutrition measurement tool developed by Ottery. ${ }^{[4]}$ The PG-SGA is an alteration of the certified nutrition assessment tool, the subjective global assessment (SGA), ${ }^{[5]}$ which has been specifically used to evaluate patients with cancer ${ }^{[6,7]}$ or chronic illness. ${ }^{[8-10]}$ This nutritional assessment tool for assessing patients with acute surgical abdomen, has seldom been used in recent medical studies.

Acute appendicitis is a common indication for abdominal surgery with a life-time incidence between $7 \%$ and $9 \%$. The incidence of acute appendicitis is greatest in the second and third decades of life, but the risk of developing acute appendicitis does not disappear at any age.$^{[11]}$ Appendectomy for acute appendicitis is one of the most common general surgical operations. ${ }^{[12]}$

Length of hospital stay (LOS) has been used as a surrogate marker of patients' well-being during hospital treatment and reflects the integration of severity of illness and patient's health status as affected by genetics, ${ }^{[13]}$ the extent or severity of the trauma or disease, overall medical or surgical treatment, quality of care, and availability of intermediary and long-term care facilities. ${ }^{[14]}$ Reducing LOS, thus, has potential to decrease health care costs, risk of infections, and other hospital-acquired diseases for improving patient quality of life. ${ }^{[13]}$

The objective of this study was to assess the validity of the scored PG-SGA as a nutritional assessment tool in adult patients who had undergone an appendectomy and to examine the association of the scored PG-SGA with LOS.

\section{METHODS}

\section{Inclusion criteria of study participants}

This retrospective study enrolled patients who had undergone an operation for suspected appendicitis at Chang Gung Memorial Hospital, Chiayi, Taiwan, between April 2010 and July 2011. The inclusion criteria of the study participants were as follows: Age ranging from 18 to 65 years, appendectomy with open procedure confined to the right lower abdominal incision (McBurney's point), and appendicitis histology confirmed by a pathologist. The Multidisciplinary Ethics Committee of the hospital approved the study (No. 100-3422B).

\section{Data collection}

Information on age, sex, body weight, body height, body mass index, C-reactive protein, hemoglobin, white blood count, LOS, Alvarado score, comorbidity defined according to Charlson scoring system, ${ }^{[15]}$ complications during hospitalization (e.g., wound infection, intra-abdominal abscess, wound hematoma and postoperative ileus), and histologic reports of an appendix were collected from patient medical records. LOS was coded as 0 for patients discharged during the day of admission. The Alvarado score is a clinical scoring system used to diagnose appendicitis. The score has 6 clinical items and 2 laboratory measurements, totaling 10 points. ${ }^{[16]}$

\section{Scored patient-generated subjective global assessment}

Nutrition assessment was conducted by experienced dietitians within 24 hours after admission by using the scored PG-SGA, anthropometric, and biochemical parameters. The scored PG-SGA comprises a medical history (weight loss, nutrition effect symptoms, intake, and functional capacity), which is completed by the patient using a check box format, as well as a physical examination, which is completed by the examiner to evaluate fat, muscle stores, and fluid status. Points were assigned for each element of the PG-SGA (0 to 4$)$, depending on the effect on nutritional status. The responses provided by participants were initially corroborated by the examiner, who subsequently yielded a global rating of well-nourished (SGA-A), moderately nourished or suspected of being malnourished (SGA-B), or severely malnourished (SGA-C). Numerical scores were allocated for each tool component and subsequently summed. Typical scores range from 0 to 35 , with a higher score reflecting a greater risk of malnutrition and scores $\geq 9$ indicating a critical need for nutrition intervention and symptom treatment. ${ }^{[4]}$

\section{Measurements}

Three measurements were conducted in this study. Regarding nutritional status, well-nourished and malnourished participants were compared. The variables potentially influencing LOS were then analyzed. The final goal is to determine the validity of the PG-SGA score in predicting the nutritional status (well-nourished vs. malnourished) of adult patients who had undergone an appendectomy.

\section{Statistical analyses}

A Chi-squared test and independent $t$ test were used to compare the categorical and continuous baseline characteristics between the well-nourished and malnourished patients. The Wilcoxon rank-sum test was used to compare the PG-SGA and Alvarado scores between the well-nourished and malnourished patients. Simple linear regression analysis 
was used to identify potential predictors for LOS. Predictors with a $p<0.10$ were entered into a multiple regression model to identify which of them significantly correlated with LOS. The Spearman rank correlation analysis was used to examine the correlation between LOS and the PG-SGA score. Receiver operator characteristic (ROC) analysis was used to examine the validity of the PG-SGA score to predict the global classifications of well-nourished or malnourished status. Stata 11 for Windows (StataCorp LP, College Station, Texas, USA) was used for statistical analyses.

\section{RESULTS}

\section{General characteristics of study participants}

A total of 172 patients had undergone an operation for suspected appendicitis in our hospital during the period from April 2010 to July 2011. Among the 172 patients, 86 patients met the inclusion criteria and 86 patients were excluded (including 20 patients confirmed with no appendicitis, 2 being opened from lower midline incision, 32 being younger than $18 \mathrm{y}, 22$ being older than $65 \mathrm{y}$, and 10 having appendicitis proven by histology but having insufficient data). The baseline characteristics of the 86 study participants are shown in Table 1 . The study included 86 patients ( 52 men and 34 women) with a mean age ( \pm SD) of $41.9 \pm 14.8$ (range, 18-65) years. The anthropometric data revealed the mean weight at $65.0 \pm 15.9 \mathrm{~kg}$ and $24.5 \pm 4.0 \mathrm{~kg} / \mathrm{m}^{2}$ for the body mass index (BMI). According to SGA classification, 71 patients $(83 \%)$ were well-nourished (SGA-A), 14 patients $(16 \%)$ were moderately nourished or suspected of being malnourished (SGA-B), and only one patient (1\%) was severely malnourished (SGA-C). Fifteen patients (17\%) were diagnosed as malnourished in this study, comprising SGA-B and SGA-C. The median (IQR) of PG-SGA scores for the 86 study participants was $3(0-5)$. The mean white blood count (WBC) was $13940 \pm 3576$ cell/uL and the median of the Alvarado score for appendicitis was 7 (6-8). The mean LOS was $4.6 \pm 2.7$ days.

\section{Comparison of variables between well-nourished and malnourished patients}

When the participants were grouped according to their SGA ratings, the adult malnourished patients who had undergone an open appendectomy were significantly older than well-nourished patients $(53.0 \pm 12.3$ years vs. $39.5 \pm 14.3$ years, $p=0.001$ ) [Table 2]. The median PG-SGA and Alvarado scores were significantly higher in the malnourished patients than in well-nourished patients ( $p<0.001$ and $p=0.028$, respectively). The number of white blood cells was slightly elevated in the malnourished patients compared with that of the well-nourished group ( $p=0.056)$. The mean LOS was longer in the mal- nourished patients $(6.9 \pm 4.2 \mathrm{~d})$ than in the well-nourished patients $(4.1 \pm 1.9 \mathrm{~d}, p=0.021)$.

The presence of comorbidity was significantly higher in the malnourished patients $(p=0.003)$ compared with the well-nourished patients. The existence of comorbidity defined in the Charlson scoring system occurred in $19(27 \%)$ of the well-nourished participants and in 10 (67\%) of the malnourished participants. The main comorbidity occurring in the well-nourished participants was mild liver disease $(37 \%)$, whereas diabetes mellitus $(50 \%)$ was the main comorbidity in the malnourished participants [Table 3].

The overall complications involved 18 events (21\%). Thirteen events in the well-nourished patients included

Table 1: Baseline characteristics for 86 adult patients receiving an open appendectomy as seen in the study

\begin{tabular}{lc}
\hline Variable & Mean \pm SD \\
\hline Age (year) & $41.9 \pm 14.8$ \\
Gender (Male:Female) & $52: 34$ \\
Weight (kg) & $65.0 \pm 15.9$ \\
Body mass index $\left(\mathrm{kg} / \mathrm{m}^{2}\right)$ & $24.5 \pm 4.0$ \\
PG-SGA Score & $3(0-5)^{*}$ \\
Nutritional Status & \\
SGA-A (well-nourished) & $71(83 \%)$ \\
SGA-B (suspected or moderately malnourished) & $14(16 \%)$ \\
SGA-C (severely malnourished) & $1(1 \%)$ \\
Alvarado score & $7(6-8)^{*}$ \\
WBC (cell/uL) & $13940 \pm 3576$ \\
Length of stay (day) & $4.6 \pm 2.7$ \\
\hline Abbreviations: SD: Standard deviation; WBC: White blood count; \\
PG-SGA: Patient generated subjective global assessment; *: Median \\
and interquartile range (IQR) for data that are not normally distributed
\end{tabular}

Table 2: Difference in measures of nutritional status in participants $(n=86)$ as seen in the study

\begin{tabular}{lccc}
\hline & $\begin{array}{c}\text { Well-nourished } \\
(n=71)\end{array}$ & $\begin{array}{c}\text { Malnourished } \\
(n=15)\end{array}$ & $p$ value \\
\hline Gender & $44(62 \%)$ & $8(53 \%)$ & $0.387^{*}$ \\
$\quad$ Men & $27(38 \%)$ & $7(47 \%)$ & \\
Women & $39.5 \pm 14.3$ & $53.0 \pm 12.3$ & $0.001^{\dagger}$ \\
Age & $65.2 \pm 16.4$ & $63.9 \pm 14.0$ & $0.745^{\dagger}$ \\
Weight $(\mathrm{kg})$ & $24.5 \pm 4.1$ & $24.3 \pm 3.7$ & $0.854^{\dagger}$ \\
Body mass index $\left(\mathrm{kg} / \mathrm{m}^{2}\right)$ & $2(0-4)$ & $8(7-10)$ & $<0.001^{\ddagger}$ \\
PG-SGA score & $7(6-8)$ & $8(7-9)$ & $0.028^{\ddagger}$ \\
Alvarado score & $64(90 \%)$ & $15(100 \%)$ & $0.205^{*}$ \\
Hi CRP (i.e., $\geq 5)$ & $13.7 \pm 1.9$ & $13.6 \pm 1.7$ & $0.732^{\dagger}$ \\
Hb & $13619 \pm 3547$ & $15570 \pm 3371$ & $0.056^{\dagger}$ \\
WBC & $19(27 \%)$ & $10(67 \%)$ & $0.003^{*}$ \\
Comorbidity & $13(18 \%)$ & $5(33 \%)$ & $0.194^{*}$ \\
Complications & $4.1 \pm 1.9$ & $6.9 \pm 4.2$ & $0.021^{\dagger}$ \\
Length of stay & & &
\end{tabular}

Abbreviations: *: Chi-squared test; ${ }^{*}$ : Independent $t$-test; *: Wilcoxon rank-sum test; WBC: White blood count; PG-SGA: Patient generated subjective global assessment; CRP: C-reactive protein; Hb: Hemoglobin 
12 wound infections and one hematoma. Five events occurred in the malnourished patients: 2 wound infections, one hematoma, one intra-abdominal abscess, and one postoperative ileus. There was no statistically significant occurrence of complication between the 2 groups. The following variables exhibited no significant difference in the well-nourished and malnourished patients: Sex, body weight, body mass index, levels of C-reactive protein, and hemoglobin.

\section{Correlation between scored patient-generated} subjective global assessment score and length of hospital stay

Simple regression analysis demonstrated that the following variables were critical risk factors for LOS: PG-SGA score $(p<0.001)$ and presence of comorbidity $(p=0.032)$ [Table 4]. The remaining variables, sex, age, body mass index, Alvarado score, white blood count, and C-reactive protein were not significant. The factors of PG-SGA score and presence of comorbidity were still significant variables in performing multiple regression analysis on LOS for adult patients who had undergone an open appendectomy ( $p=0.001$ and $p=0.044$, respectively). Correlation analysis revealed a significantly positive correlation between LOS and PG-SGA score (Spearman's $r h o=0.378, p<0.001)$ [Figure 1].

Table 3: The occurrence of comorbidity in well-nourished and malnourished patients as seen in the study

\begin{tabular}{lcccc}
\hline & $\begin{array}{c}\text { Well-nourished } \\
(n=71)\end{array}$ & \multicolumn{2}{c}{$\begin{array}{c}\text { Malnourished } \\
(n=15)\end{array}$} \\
\hline Mild liver disease & 7 & $(37.0 \%)$ & 2 & $(16.7 \%)$ \\
Peptic ulcer disease & 4 & $(21.1 \%)$ & 2 & $(16.7 \%)$ \\
Chronic pulmonary disease & 1 & $(5.3 \%)$ & 1 & $(8.3 \%)$ \\
Diabetes mellitus & 3 & $(15.7 \%)$ & 6 & $(50.0 \%)$ \\
Renal disease & 1 & $(5.2 \%)$ & 0 & $(0 \%)$ \\
Rheumatological disease & 3 & $(15.7 \%)$ & 0 & $(0 \%)$ \\
Dementia & 0 & $(0 \%)$ & 1 & $(8.3 \%)$ \\
Total & 19 & & $12 *$ & \\
\hline
\end{tabular}

*: Two patients had two comorbidities

Table 4: Regression analysis on length of stay as seen in the study

\begin{tabular}{lccccc}
\hline & \multicolumn{2}{c}{ Simple regression } & & \multicolumn{2}{c}{ Multiple regression } \\
\cline { 2 - 3 } Sex & Coefficient & $p$ value & & Coefficient & $p$ value \\
Age & 0.718 & 0.225 & & \\
Body mass index & 0.024 & 0.221 & & \\
PG-SGA score & 0.063 & 0.384 & & \\
Alvarado score & 0.313 & $<0.001$ & & 0.300 & 0.001 \\
Comorbidity & 0.232 & 0.196 & & \\
CRP $\geq 5$ & 1.513 & 0.032 & & 1.334 & 0.044 \\
Leukocytosis* & -0.300 & 0.778 & & \\
\cline { 1 - 2 } & 0.667 & 0.443 & & \\
\hline
\end{tabular}

Abbreviations: *: Leukocytosis: WBC $>10.6 \times 10^{3} / \mathrm{uL}$ in men and

$>11.0 \times 10^{3} / \mathrm{uL}$ in women; PG-SGA: Patient generated subjective global assessment; CRP: C-reactive protein

\section{Validity of scored patient-generated subjective global assessment score and receiver operator characteristic curve}

Based on the triage recommendations of Ottery, ${ }^{[4]}$ a PG-SGA score between 4 and 8 points indicates the need for dietitian intervention in conjunction with a nurse or physician, as indicated by symptoms; a PG-SGA score $\geq 9$ points indicates the need for critical nutritional intervention; thus, the validity of the PG-SGA score in predicting the global classifications of well-nourished or malnourished patients was determined. The ROC curve depicting the cross-validity of the PG-SGA score is presented in Figure 2. The area under the ROC curve for the PG-SGA score compared with nutritional status (well-nourished vs. malnourished) is 0.9751 , showing high accuracy according to an arbitrary standard. ${ }^{[17,18]}$ The cutoff value of 7 points using the scored PG-SGA had the highest accuracy (97.67\%) in this analytic model. The sensitivity and specificity of the PG-SGA scores for nutritional status were $86.67 \%$ and $100 \%$, respectively.

\section{DISCUSSION}

To the best of our knowledge, this study was the first to evaluate the nutritional status of patients with acute appendicitis by using the scored PG-SGA. Numerous previous studies have used the PG-SGA score to assess the nutritional status of patients with chronic illness ${ }^{[9,10,19,20]}$ and cancer, ${ }^{[21-24]}$ rather than assessing patients with acute illness, in contrast to the current study. We determined the nutritional status of adult patients who had undergone an appendectomy by using the scored PG-SGA and assessed the associations

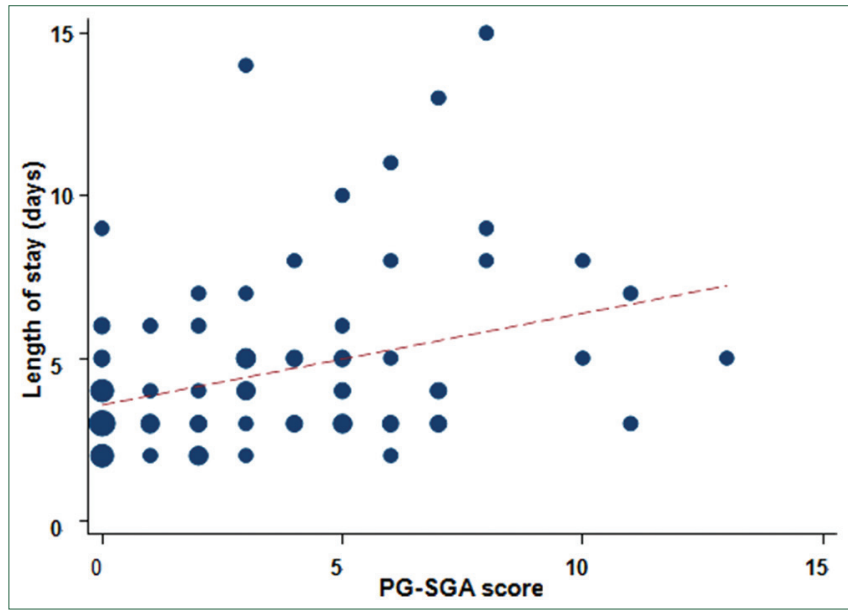

Figure 1: Correlation of scored patient-generated subjective global assessment (PG-SGA) score with length of stay in 86 participants. The area of spot is proportional to the participants. Correlation analysis was performed using the Spearman correlation test $(r h o=0.378$, $p<0.001, n=86$ ) 


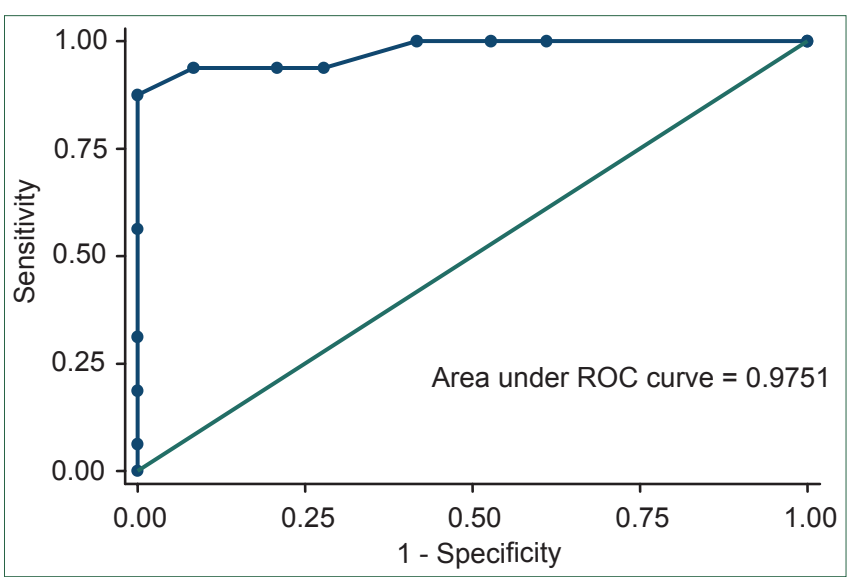

Figure 2: Receiver operator characteristic (ROC) curve for scored patient-generated subjective global assessment (PG-SGA) for adult patients who had undergone an open appendectomy compared with nutritional status (malnourished vs. nourished). The $45^{\circ}$ line represents a curve for an ROC area of 0.5 . The area under the curve (AUC) is 0.9751, thus indicating the PG-SGA score

of nutritional status with health outcomes, such as LOS, complications, and comorbidities.

Acute appendicitis is a common cause of abdominal surgery with a life-time incidence between $7 \%$ and $9 \%,{ }^{[11]}$ and an appendectomy is one of the most common surgical procedures. Adults with acute appendicitis receiving an open appendectomy were the inclusion criteria in this study. Some previous studies have suggested that appendicitis in elderly people ${ }^{[25,26]}$ and children ${ }^{[27]}$ is commonly associated with delayed diagnosis or atypical presentation, a high rate of perforation and complications, and increased LOS. A meta-analysis of randomized controlled trials showed that a laparoscopic appendectomy provides considerable benefits over an open procedure, including a shorter hospital stay, less postoperative pain, and a lower complication rate. ${ }^{[28]}$ Based on both of these reasons, this study established limit to reduce the selection bias and improve the accuracy of the results.

According to the categorical grouping of SGA, 71 patients $(83 \%)$ were well-nourished and $15(17 \%)$ were malnourished. The incidence of malnutrition was relatively low in this study compared to those that have examined patients with cancer, ${ }^{[8]}$ chronic kidney disease, ${ }^{[9]}$ and stroke, ${ }^{[10,19]}$ which have ranged from $19.2 \%$ to $74 \%$. This is possibly because the participants in this study had acute illness instead of chronic illness or cancer. Only one studyon chronic pulmonary disease had lower incidence $(15.2 \%),{ }^{[20]}$ and the authors of that study considered a higher socioeconomic status of the participants to be the main cause.

The malnourished participants in the current study had significantly higher (median, IQR) PG-SGA scores $(8,7-10)$ compared with the well-nourished participants $(2,0-4)$. This finding agrees with studies that have used this screening tool to evaluate patients with cancer, ${ }^{[7,8,20]}$ chronic kidney disease, ${ }^{[9]}$ and stroke. ${ }^{[10]}$ The scored PG-SGA yields both a qualitative categorization (well-nourished, moderately nourished or suspected of being malnourished, and severely malnourished) and a quantitative score. The categorization can be used to determine the nutritional status of patients and the score can be used to triage nutrition intervention, ${ }^{[4]}$ as an outcome measure to demonstrate change in nutritional status, ${ }^{[29]}$ or as a surrogate measure of change in quality of life. ${ }^{[30]}$ The numerical score is more sensitive to smaller changes in nutritional status than the global categorization. ${ }^{[29]}$

Compared to the well-nourished patients, the malnourished patients were of significantly older age $(p=0.001)$, even though we limited the age of included participants to between 18 and 65 years. The Alvarado score was used to assess the findings of several clinical and laboratory studies to identify the patients who were suspected of appendicitis and required surgical management. ${ }^{[31]}$ This study found that the malnourished participants had a significantly higher Alvarado score compared to the well-nourished participants $(p=0.028)$. The higher the Alvarado scores are, the higher the SGA rating is. This is because some items assessed in the Alvarado score exist in SGA categorization, such as abdominal pain, nausea, vomiting, and anorexia. In other words, malnourished patients might have weaknesses in their immune response to infections.

Comorbidity has been proven as an independent determinant of costs and LOS in surgical patients. ${ }^{[32]}$ The critical variable of a higher comorbidity rate occurred in the malnourished group (67\% vs. $27 \%, p=0.003$ ). Diabetes mellitus was the major comorbidity in the malnourished patients, whereas mild liver disease was the major comorbidity in the well-nourished patients. The presence of comorbidity was a slightly significant risk factor for LOS for adult patients receiving an appendectomy after regression analysis $($ coefficient $=1.334, p=0.044)$. This might be because malnourished patients easily contract a chronic illness, such as diabetes mellitus, peptic ulcer disorder, rheumatologic disease, chronic kidney, or liver and pulmonary disease, thus compromising their defense system to conquer acute insult and prolonging LOS. The other variables of sex, body weight, body mass index, C-reactive protein level, hemoglobin, white blood count, and surgical complications were not significant in this study.

LOS has been used as a surrogate marker of patient well-being during hospital treatment. ${ }^{[13]}$ Reducing the LOS has the potential to decrease health care costs, risk of infections, and to improve patient quality of life. ${ }^{[13]}$ The data revealed that malnourished patients had significantly longer LOS $(6.9 \pm 4.2)$ compared to well-nourished patients $(4.1 \pm 1.9, p=0.021)$. The factor of PG-SGA 
score was the significant predictors for LOS after simple and multiple regression analysis (coefficient $=0.300$, $p=0.001$ ) [Table 4]. A significantly positive correlation existed between PG-SGA score and LOS ( $r h o=0.378$, $p<0.001$ ) [Figure 1]. The possible reason was the participants with higher PG-SGA score might present with more nutrition effect symptoms, such as fever, poor appetite, vomiting, diarrhea or abdominal pain. These unfavorable events could compromise the participants' ability to overcome the acute surgical impacts, then getting more surgical complications or delaying postoperative recovery which resulted in longer LOS. Horsley et al., reported nutritional status as determined by PG-SGA score to be significantly associated with LOS for peripheral blood stem cell transplantation participants. ${ }^{[33]}$ Laky et al., considered the PG-SGA score to be a critical variable for predicting LOS in univariate analysis, but did not deem the PG-SGA score significant in the multivariate model for gynecological cancer patients. ${ }^{[13]}$ Based on these results, the PG-SGA score was determined to be a significant predictor of LOS, in that the mean LOS was significantly lower in the well-nourished group versus that of the malnourished group among cancer patients and adult patients receiving an open appendectomy. The limitation of this study is the severity of acute appendicitis not examined whether affected LOS, although longer LOS in participants with complicated appendicitis has been well-known. ${ }^{[34]}$

The validity of the PG-SGA score with nutritional status has been measured from 2 aspects. Ottery ${ }^{[4]}$ recommended that triage nutrition intervention be based on the PG-SGA score, which requires involvement by a dietitian, nurse, and physician when the score is from 4 to 8 ; and a PG-SGA score $\geq 9$ indicates the need for critical nutrition intervention options. The results showed that the area under the ROC curve is 0.9751 , with a sensitivity of $86.7 \%$ and a specificity of $100 \%$ for predicting nutritional status (ie, malnourished or well-nourished), when the cutoff value of the PG-SGA score is 7. Laky et al., ${ }^{[23]}$ reported that the PG-SGA score is a significant parameter for predicting the nutritional status of gynecological cancer patients and the AUC is 0.92 . In patients with chronic obstructive pulmonary disease, the PG-SGA score is an effective nutrition assessment tool and has a sensitivity of $82 \%$ and a specificity of $79 \%$ if the PG-SGA score is $\geq 9$. Using the PG-SGA score to predict nutritional status of adult patients who had undergone an open appendectomy is superior to the methods used in the previous 2 studies. The likely reason is due to the participants in this study belonged to acute disease that upset within a couple days, rather than those who suffered from chronic illness or cancer for a longer period. A significantly positive correlation also existed between the PG-SGA score and LOS. This emphasizes the advantage of using the PG-SGA score to forecast LOS, which might reduce health care costs, lower the risk of infections, and improve patient well-being.

In summary, malnutrition in adults receiving an open appendectomy is associated with prolonged LOS.The scored PG-SGA is an effective tool for assessing the nutritional status of patients with cancer and chronic illness, as well as of patients with acute surgical abdomen.

\section{ACKNOWLEDGEMENTS}

The authors are indebted to the dietitians at the Department of Nutrition for data collection.

\section{REFERENCES}

1. Correia MI, Waitzberg DL. The impact of malnutrition on morbidity, mortality, length of hospital stay and costs evaluated through a multivariate model analysis. Clin Nutr 2003;22:235-9.

2. Corish CA, Kennedy NP. Protein-energy malnutrition in hospital in-patients. Br J Nutr 2000;83:575-91.

3. Green CJ. Existence, causes, and consequences of disease-related malnutrition in the hospital and the community, and clinical and financial benefits of nutrition intervention. Clin Nutr 1999;18(Suppl):3-28.

4. Ottery F. Patient-generated subjective global assessment. In: McCallum P, Polisena C (editors). The Clinical Guide to Oncology Nutrition. Chicago, IL: American Dietetic Association; 2000. p. 11-23.

5. Detsky AS, McLaughlin JR, Baker JP, Johnston N, Whittaker S, Mendelson RA, et al. What is subjective global assessment of nutritional status? J Parenter Enteral Nutr 1987;11:8-13.

6. Janda M, Obermair A, Laky B, Cleghorn G. Comparison of different nutritional assessments and body-composition measurements in detecting malnutrition among gynecologic cancer patients. Am J Clin Nutr 2008;87:1678-88.

7. Chasen MR, Bhargava R. A rehabilitation program for patients with gastroesophageal cancer: A pilot study. Support Care Cancer 2010;18:35-6.

8. Isenring E, Thomas JM, Kellett E. Nutritional status and length of stay in patients admitted to an acute assessment unit. J Hum Nutr Dietetics 2007;20:320-9.

9. Desbrow B, Bauer J, Blum C, Kandasamy A, McDonald A, Montgomery K. Assessment of nutritional status in hemodialysis patients using patient-generated subjective global assessment. J Ren Nutr 2005;15:211-6.

10. Martineau J, Bauer JD, Isenring E, Cohen S. Malnutrition determined by the patient-generated subjective global assessment is associated with poor outcomes in acute stroke patients. Clin Nutr $2005 ; 24: 1073-7$

11. Addiss DG, Shaffer N, Fowler BS, Tauxe RV. The epidemiology of appendicitis and appendectomy in the United States. Am J Epidemiol 1990;132:910-25.

12. DeFrances CJ, Cullen KA, Kozak LJ. National Health Discharge Survey: 2005 annual summary with detailed diagnosis and procedure data. Vital Health Stat 2007;13:1-209.

13. Laky B, Janda M, Kondalsamy-Chennakesavan S, Cleghorn G, 
Obermair A. Pretreatment malnutrition and quality of life-association with prolonged length of hospital stay among patients with gynecological cancer: A cohort study. BMC Cancer 2010;10:232.

14. Kyle UG, Genton L, Pichard C. Hospital length of stay and nutritional status. Curr Opin Clin Nutr Metab Care 2005;8:397-402.

15. Charlson ME, Pompei P, Ales KL, MacKenzie CR. A new method of classifying prognostic comorbidity in longitudinal studies: Development and validation. J Chronic Dis 1987;40:373-83.

16. Alvarado A. A practical score for the early diagnosis of acute appendicitis. Ann Emerg Med 1986;15:557-65.

17. Greiner M, Pfeiffer D, Smith RD. Principles and practical application of the receiver-operating characteristic analysis for diagnostic tests. Prev Vet Med 2000;45:23-41.

18. Swets JA. Measuring the accuracy of diagnostic systems. Science 1988;240:1285-93.

19. Lim HJ, Choue R. Nutritional status assessed by the patient-generated subjective global sssessment (PG-SGA) is associated with qualities of diet and life in Korean cerebral infarction patients. Nutrition 2010;26:766-71.

20. Bauer J, Egan E, Clavarino A. The scored patient-generated subjective global assessment is an effective nutrition assessment tool in subjects with chronic obstructive pulmonary disease. European e-journal of clinical nutrition and metabolism 2011;6:e27-e30.

21. Kim JY, Wie GA, Cho YA, Kim SY, Kim SM, Son KH, et al. Development and validation of a nutrition screening tool for hospitalized cancer patients. Clin Nutr 2011;30:724-9.

22. Isenring E, Bauer J, Capra S. The scored patient-generated subjective global assessment (PG-SGA) and its association with quality of life in ambulatory patients receiving radiotherapy. Eur J Clin Nutr 2003;57:305-9.

23. Laky B, Janda M, Cleghorn G, Obermair A. Comparison of different nutritional assessments and body-composition measurements in detecting malnutrition among gynecologic cancer patients. Am J Clin Nutr 2008;87:1678-85.
24. Hill A, Kiss N, Hodgson B, Crowe TC, Walsh AD. Associations between nutritional status, weight loss, radiotherapy treatment toxicity and treatment outcomes in gastrointestinal cancer patients. Clin Nutr 2011;30:92-8.

25. Storm-Dickerson TL, Horattas MC. What have we learned over the past 20 years about appendicitis in the elderly? Am J Surg $2003 ; 185: 198-201$

26. Hui TT, Major KM, Avital I, Hiatt JR, Margulies DR. Outcome of elderly patients with appendicitis: Effect of computed tomography and laparoscopy. Arch Surg 2002;137:995-8.

27. Narsule CK, Kahle EJ, Kim DS, Anderson AC, Luks FI. Effect of delay in presentation on rate of perforation in children with appendicitis. Am J Emerg Med 2011;29:890-3.

28. Li X, Zhang J, Sang L, Zhang W, Chu Z, Li X, et al. Laparoscopic versus conventional appendectomy-a meta-analysis of randomized controlled trials. BMC Gastroenterol 2010;10:129.

29. Bauer JD, Capra S. Nutrition intervention improves outcomes in patients with cancer cachexia receiving chemotherapy: A pilot study. Support Care Cancer 2005;13:270-4.

30. Isenring EA, Capra S, Bauer JD. Nutrition intervention is beneficial in oncology outpatients receiving radiotherapy to the gastrointestinal or head and neck area. Br J Cancer 2004;91:447-52.

31. Andersson M, Andersson RE. The appendicitis inflammatory response score: A tool for the diagnosis of acute appendicitis that outperforms the Alvarado score. World J Surg 2008;32:1843-9.

32. Cho JY, Han HS, Yoon YS, Ahn KS. Risk factors for acute cholecystitis and a complicated clinical course in patients with symptomatic cholelithiasis. Arch Surg 2010;145:329-33.

33. Horsley P, Bauer J, Gallagher B. Poor nutritional status prior to peripheral blood stem cell transplantation is associated with increased length of hospital stay. Bone Marrow Transplant 2005;35:1113-6.

34. Ingraham AM, Cohen ME, Bilimoria KY, Ko CY, Hall BL, Russell TR, et al. Effect of delay to operation on outcomes in adults with acute appendicitis. Arch Surg 2010;145:886-92. 